\title{
Therapy of melanocytic conjunctival tumors
}

\author{
Halas M Jr' ${ }^{1}$, Svetlosakova $\mathrm{Z}^{1}$, Babal $\mathrm{P}^{2}$ \\ Department of Ophthalmology, Comenius University, Medical Faculty, University Hospital, \\ Bratislava,Slovakia.marianhalas@gmail.com
}

\begin{abstract}
Background: Clinical experience of our single center in dealing with pigmented epibulbar lesions melanocytic conjunctival tumors is presented. Since 2008 we use the topical treatment with mitomycin C (MMC) as an alternative or adjunctive method for excision in the treatment of melanocytic neoplasia of the conjunctiva. Methods: The retrospective case series of 85 patients with pigmented lesions of the conjunctiva - melanocytic conjunctival tumors, histopathologically examined in the period 2001-2010 is presented. Since 2008 we started to apply MMC in the treatment of primary acquired melanosis (PAM) and dysplastic nevi. We apply MMC topically directly after an excision as 2-times five minutes application.

Results: In 85 patients with pigmented lesions of the conjunctiva histopathological findings after excision of the lesion showed in 68 (80 \%) cases melanocytic nevocelullar nevus, out of which 55 cases were combined and 13 cases were junctional nevi. In $60(80 \%)$ cases of melanocytic nevi atypia was found in 25 patients $(42 \%)$, nevus without atypia was present in 35 cases (58\%). PAM with atypia was found in 16 patients (classified since 2000). During the period of application of MMC we diagnosed only one patient with primary conjunctival melanoma. There was no presence of relapse of the pigmented lesion either after primary excision or after excision with MMC.

Conclusion: Resection of more than one quadrant of bulbar conjunctiva in patients with pigmented lesions of the conjunctiva in cases of conjunctival nevus with atypia and PAM with atypia combined with topical MMC chemotherapy is an alternative therapy for residual pigmented lesions. There was no presence of relapse of pigmentation in area of excision with or without using MMC during the surgery in patients with PAM. The number of our patients is not sufficient yet to draw a conclusion (Fig. 6, Ref. 21). Full Text in PDF www.elis.sk. Key words: conjunctival neoplasia, primary acquired melanosis, mitomycin $\mathrm{C}$ therapy.
\end{abstract}

Melanocytic lesions of the ocular adnexa include nevi, primary acquired melanosis of the conjuctiva (PAM) with or without atypia, and malignant melanoma (MM). The classification divides them in congenital and acquired lesions, which are generally unilateral. Conjunctival nevus is the most common type of benign tumors of the conjunctiva. This lesion is usually congenital, with the highest incidence in the second and third decade of life if they are pigmented (1). Conjunctival melanoma has an incidence of less than one per million per year (2) and represents only $2 \%$ of all malignant diseases of the eye. It is lethal neoplasm, with an average 10-year mortality rate of $30 \%$. Melanoma of the conjunctiva tend to present in adulthood, especially in caucasion population. The median age at diagnosis is 60 years (3).

Melanoma arises from three sources-PAM, nevus, or de novo. Approximately $75 \%$ of cases have PAM, up to $20 \%$ of patients have history or microscopic evidence of a benign nevus and $5 \%$ are de novo melanomas. Melanomas of the conjunctiva are deeply pigmented, lightly pigmented or amelanotic. Their margins may be discrete or diffuse. They can be unifocal or multifocal (3). Side effects of

${ }^{1}$ Department of Ophthalmology, Comenius University, Medical Faculty, University Hospital, Bratislava, and ${ }^{2}$ Institute of Pathology, Comenius University, Medical School

Address for correspondence: M. Halas, MD, Sancova 54, SK-81105 Bratislava, Slovakia.

Phone: +421.903574784 this tumor are dilated feeder vessels and degenerative changes of the cornea when the tumor involves cornea (3). Histopathology of these lesions shows a loss of normal polarity. The cells are atypical, with an increased nuclear to cytoplasmic ratio and scattered mitoses (4).

Clinical examination findings involve discrete well defined congenital lesions that are most commonly found on the interpalpebral bulbar conjunctiva. These lesions can also involve the caruncle, plica, and lid margin (5). They are rarely found in the fornix.

Histopathologic findings show benign proliferation of nevus cells present predominantly in the substantia propria. Histologically, PAM is characterized by melanocytes in the vicinity of the basement membrane of the epithelium with or without atypical cells and amplified pigmentation. In PAM without atypia, there is almost never a malignant transformation $(6,7)$.

Treatment involves either observation or local excision if the lesion is unsightly or shows unusual growth (8). The treatment is necessary to reduce the possibility of spread of the tumor. Melanoma of the conjunctiva spreads into the regional lymphatics. The median time of regional and systemic metastases is about 2 years, the risk of MM of the conjunctiva within 10 years is up to $30 \%$ (9). All suspicious lesions should be histologically examined to confirm the clinical diagnosis (10).

Risk factors for metastasis include more than $2 \mathrm{~mm}$ in thickness, the participation of nonbulbar conjunctiva, caruncular disease, medial tumor location and tumor recurrence (3). Therefore, local exci- 


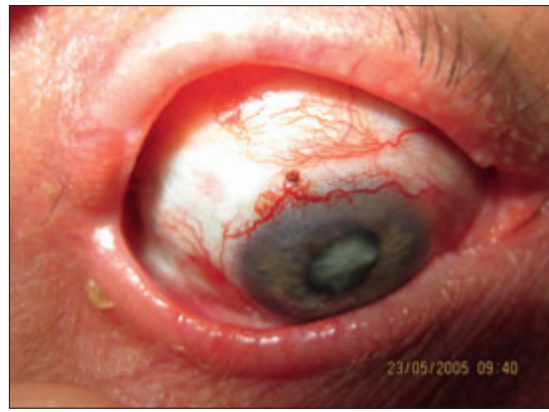

Fig. 1a. Patient, male, 65 years old with pigmented lesion of the conjunctiva 16 months m after stereotactic radiosurgery at linear accel-
erator due to malignant choroidal melanoma. (Department of Ophthalmology, Bratislava).

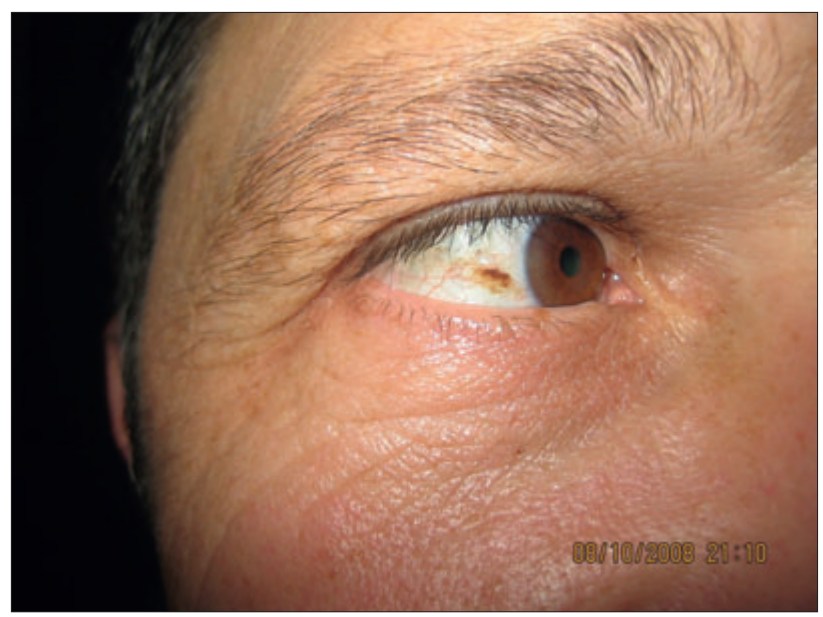

Fig. 2. Patient, male, 47 years old with a conjunctival nevus cyst. (Department of Ophthalmology, Bratislava).

sion with a „no-touch technique“ is the goal of treatment. Diffuse disease can be treated by cryotherapy or topical adjuvant chemotherapy with mitomycin C. If the disease is too extensive for ocular conservation, exenteration is performed. Some authors advocate sentinel lymph node biopsy in high-risk cases [3]. Good results in tumor control can be achieved by brachytherapy (11). Latest trends in therapy with interferon alfa2b were reported by the PAM (12).

Mitomycin C (MMC) is originally an antibiotic from Streptomyces caespitosus and belongs to the group of alkylating agents and inhibits DNA synthesis. MMC reacts under aerobic conditions with molecular oxygen and intermediates are produced. These in turn are known for their cytotoxic effects of lipid peroxidation. Mitomycin $\mathrm{C}$ is used in ophthalmology for many years to prevent recurrence of pterygium and in conventional glaucoma surgery. Ocular use of topical MMC range from prevention of pterygium recurrence in the treatment to prevent transformation to squamous neoplasia of the conjunctiva (13).

\section{Material and methods}

Retrospective study of the records from 85 patients examined histopathogically for pigmented lesions of the conjunctiva in the

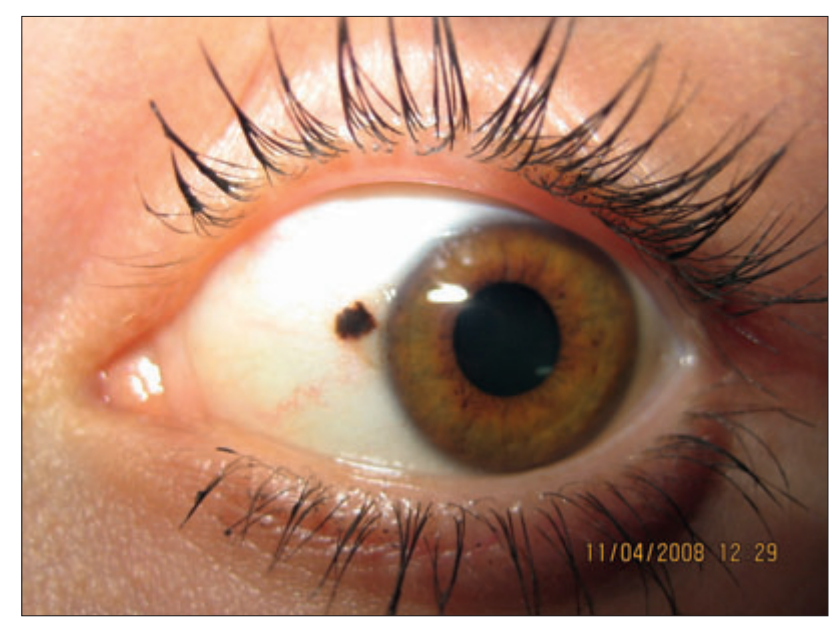

Fig. 3. Patients, female, 51 years old with perilimbal nevus. (Department of Ophthalmology, Bratislava).

period 2001 to 2010 at the Department of Ophthalmology, Comenius University, Medical School, Bratislava was performed. In all cases, primary excision was the first step in the process. The indications for excision and histopathological examination were changes in color (darkens gradually) and the shape of the pigmented mass in patients who returned for periodic observation (3 months interval). Primary indication for surgery was localization of the nevus on the limbus less than $0.5 \mathrm{~mm}$ from the edge of the cornea in patients who wanted to correct refractive error before starting to wear their contact lenses. Each patient after excision was asked to come for a control visit at least every 12 months. Histopathological evaluation was performed using light microscopy after routine fixation in $10 \%$ formalin and processed in paraffin. The $5 \mathrm{~mm}$ thick sections were stained with hematoxylin and eosin.

We started using MMC therapy in 2008. The therapy was administered to patients after primary excision. Treatment of the MMC is still off-label in our conditions. We used MMC $(0.04 \%=$ $0.4 \mathrm{mg} / \mathrm{l})$ as a topic treatment after excision two times for $5 \mathrm{~min}$ in the operating room. The patients did not receive MMC fluid for use at home. We have used only one concentration of MMC. Followup was performed 2 weeks, 6 weeks and then in 3-month intervals 

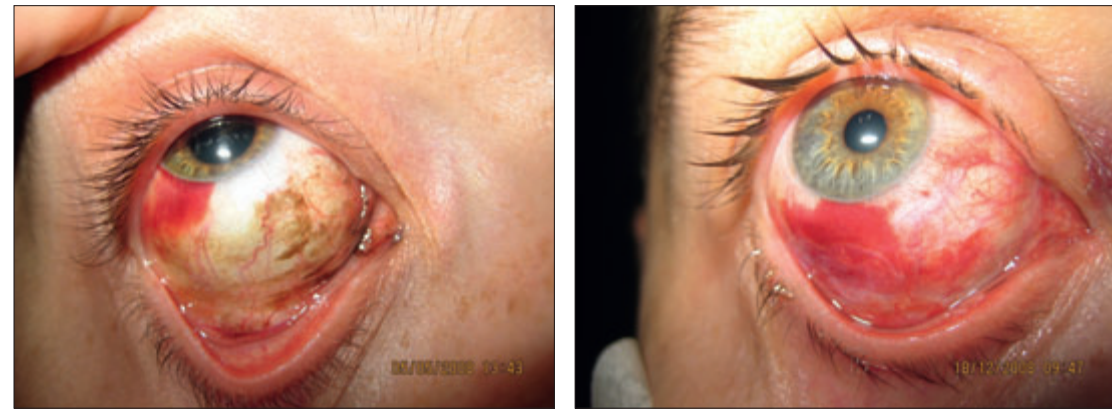

Fig. 4a. Patient, female, 45 years old with PAM
with atypia before the therapy - May 2008. (Department of Ophthalmology, Bratislava).
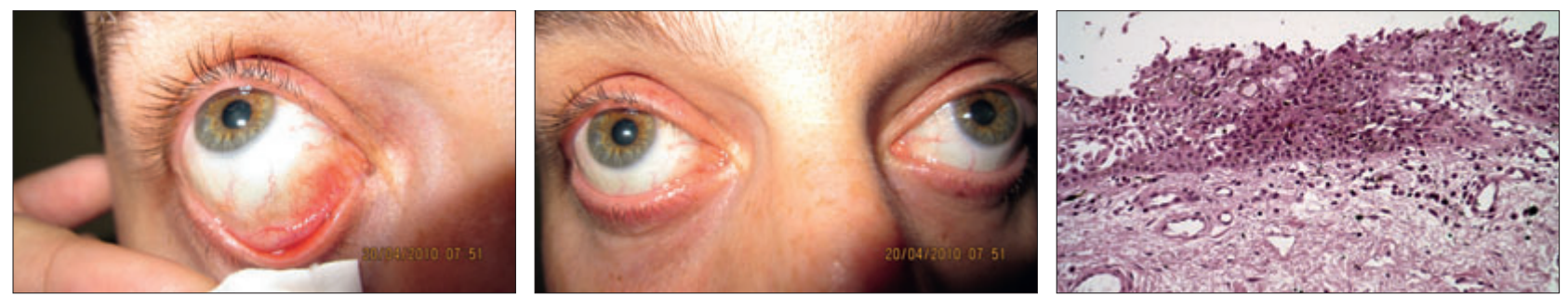

Fig.4d.Patient 2 yearsafter treatment-April2010. Fig. 4e. Patient 2 years after therapy (both eyes) Fig. 4f. Histopathological findings before therapy (Department of Ophthalmology, Bratislava). (Department of Ophthalmology, Bratislava).

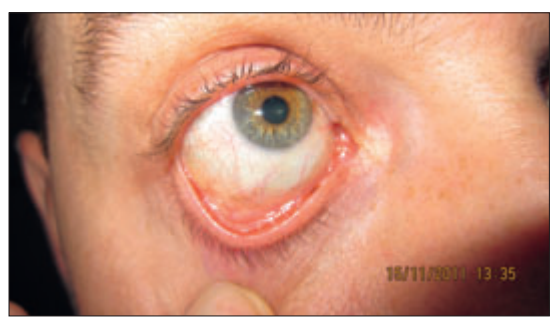

Fig. 4g. Patient 3 years after treatment (2011) (Institute of Pathology, Bratislava).

in the first year, later in 12 month intervals (slit lamp examination, photo documentation, visual acuity, intraocular pressure).

\section{Results}

We examined 85 patients with pigmented lesions - melanocytic conjunctival tumors of the ocular surface, 47 (55\%) male and 38 (45\%) female. We excluded other pigmented lesions like dark foreign bodies, racial melanosis or pigmented pinguecula. Average age was 46 years (14 to 79 years). All patients with epibulbar pigmented lesions - brown, dark brown and semi-pigmented lesions were involved.

Unilateral lesions were found in 64 (75\%) cases. In 84 (99\%) cases we found nevi or primary acquired melanosis of the conjunctiva and only 1 case of malignant melanoma was histologically verified. In 85 patients with pigmented lesions of conjunctiva after excision in 68 cases melanocytic nevi $(80 \%)$ were found. Primary acquired melanosis with atypia was found in 16 (19\%) cases.

One patient with a pigmented lesion, which grew out through the sclera in the event of the verified intraocular uveal melanoma

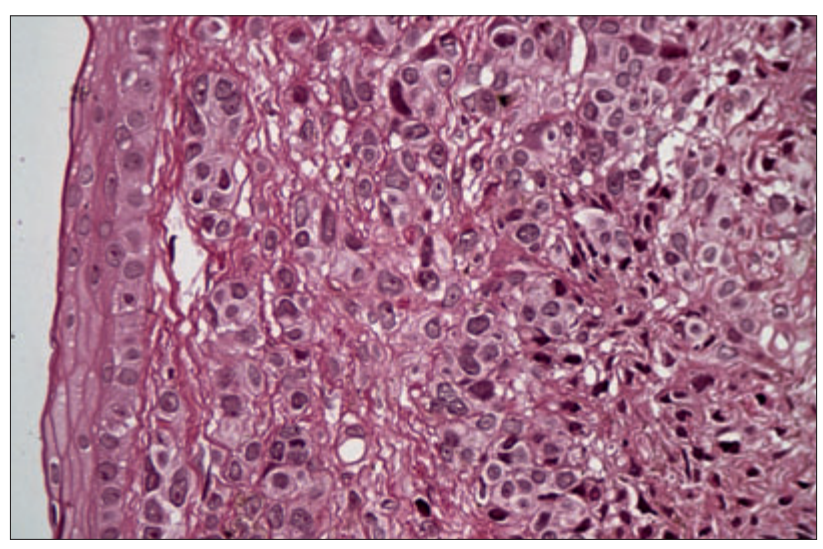

Fig. 5. Subepithelial nevus without nodular dysplasia (HE, 200x) (Department of Ophthalmology, Bratislava).

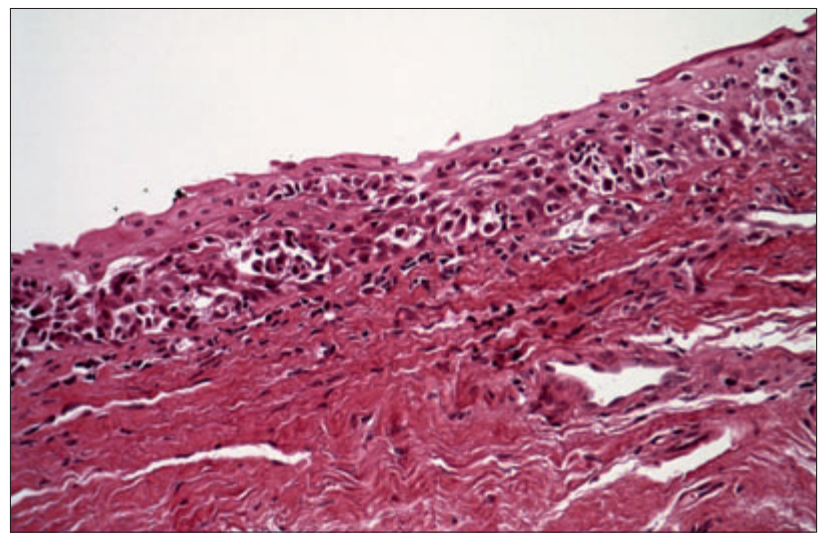

Fig. 6. Nevus of the conjunctiva (HE, 100x). 
after combined therapy (radiosurgery on linear accelerator using therapeutic dose of 35.0 Gy and endoresection) was histopathologically classified as PAM with atypia (Figs 1 a, b, c). The most common sites were nevus on the bulbar conjunctiva -69 cases $(81 \%)$, caruncle -7 cases $(8 \%)$ and plica semilunaris -8 cases $(9$ $\%)$. In the patient with melanoma this was verified from primary lesion arising form caruncle. Nevi were seen most frequently in the nasal and temporal parts of the conjunctiva (over $75 \%$ ). Intralesional cysts were present in 48 patients $(57 \%)$ - they were present clinically - significant by slit lamp examination (Figs 2 and 3).

In patients with PAM in 7 cases the lesion was spread more than in one quadrant, and in one case, the infiltration of the fornix and tarsal conjunctiva was present. MMC was used peroperatively after excision of the lesion. In one patient with PAM with atypia localised in more than 2 quadrants and plica and caruncle we applied MMC in three sessions in 3-month interval (Figs 4 a, b, c, d, e, f, g). In patients with nevi without dysplasia (Figs 5 and 6) MMC was not used. There was no presence of relapse of pigmentation in the area of excision with or without using MMC during the surgery in patients with PAM.

The observation interval ranged from 1 year up to 8 years, we did not find relapse of the primary pigmented lesion in our patients.

\section{Discussion}

Primary acquired melanosis is a potentially serious melanocytic lesion leading to the development of melanoma (14). PAM can preceed $11 \%$ of all tumors of the conjunctiva and $21 \%$ of melanocytic skin lesions (7). PAM presents clinically as a unilateral, superficial, flat, brown discoloration of the conjunctiva, most commonly affects middle-aged or elderly Caucasian patients. It is necessary to differentiate PAM from more benign conditions such as nevi, racial and secondary melanosis. Unlike PAM, racial melanosis is a bilateral disease and occurrs in more pigmented races. This benign condition has little malignant potential and does not tend to spread to the cornea. Melanosis of the conjunctiva can be secondary to metabolic disorders such as Addison's disease, pregnancy or can be caused by toxic effects of drugs such as arsenic, chlorpromazine, epinephrine. Diseases, chronic inflammation and scarring, such as trachoma or vernal conjunctivitis, can also produce increased postinflammatory pigmentation.

The reason why PAM attracts so much attention is that it can be turned to the potential malignant melanoma of the conjunctiva. Unfortunately it is impossible to predict the clinical lesions with the highest probability of malignant transformation. The risk of malignant change depends on the microscopic properties of the biopsies.

The terminology associated with melanosis is controversial. The term PAM with or without atypia is the histological features of these lesions (5). In PAM without atypia appearing melanocytes colonize the epithelium, whereas PAM with atypia is characterized cytologically by abnormal melanocytes identified subtypes, such as polyhydral cells, spindle cells, large cell or epitheloid dendritic form cells. The abnormal cells that can characterize PAM with atypia should be limited to the basilar region, or spread to all levels of the epithelium. PAM without atypia appears to have mini- mal malignant potential, whereas PAM with atypia can progress to malignant melanoma in half of the cases (15). The median time to progression of PAM with atypia to melanoma is approximately 2.5 years, but it can be as long as 10 years (6). The main goal of treatment of this disease is to prevent the invasive conjunctival melanoma. There is a tendency to observe small lesions and to treat more extensive disease. Some experts advocate excision and cryotherapy for larger lesions that are associated with greater risks. An amniotic membrane or buccal mucosa graft is used after extensive excision. Residual disease can be treated with cryotherapy and/ or topical chemotherapy with mitomycin C drops (14).

The main objective of management of pigmented lesions, is to avoid the transformation to invasive melanoma of the conjunctiva. There is a tendency to observe small lesions and to treat more extensive disease.

PAM with atypia compares the success rate of topical MMC favorably with the recurrence rate that was reported after local excision and cryotherapy. Therefore, topical MMC can be considered a viable therapeutic option for patients with diffuse PAM with atypia or in patients with positive margins or residual areas of pigmentation that are diagnosed following local excision. In most protocols MMC of $0.04 \%$ has been used four times daily for 2 week cycles, with intervals of 1-2 weeks between cycles, and a total of 2-4 cycles to allow for a complete clinical response. It should be emphasized that not all areas of pigmentation resolve completely, and a clinical response can be continued to be observed for months after the topical treatment was stopped (10).

For invasive melanoma of the conjunctiva, the role of topical MMC in the treatment algorithm is not well established. If a melanoma of the conjunctiva is removed surgically and the surgical margins show an area of PAM with atypia or residual superficial disease of the cornea, a topical therapy may be repeated as a viable alternative to surgical excision. If the surgical margin is positive for invasive melanoma, surgical excision of the affected area should be seriously considered (16).

The clinical features of pigmented lesions of the conjunctiva can sometimes overlap most reports from data on the histopathological features of conjunctival nevi provided. The frequency of nevi involving the tarsal conjunctiva or fornix support the idea that pigmented lesions - nevi are not presnet in these areas and that the other conditions, such as conjunctival melanoma, should be considered. The majority of conjunctival nevi (more than $60 \%$ ) in the series contained cysts, which are not seen in the conjunctival melanoma (5).

Primary melanoma of the conjunctiva is a condition of concern because of its rarity and lethal potential. About $75 \%$ of these tumors arise in an area of PAM with atypia that requires histopathologic examination for the diagnosis. If thickening of the conjunctiva is in an area of PAM currently developing invasive malignant melanoma is potential. According to previous study results with MMC in treatment of pigmented lesions of the conjunctiva positive results should be confirmed in large multicenter studies with long-term follow $(10,17,18)$.

The recognition of the precursor of melanoma at an early stage is important. Staging of the disease by sentinel lymph node bio- 
psy is now recommended in some centers. The surgical excision with adjuvant cryotherapy and alcohol corneal epithelialectomy is usually effective in eradicating most of these lesions. Multifocal and advanced melanoma are managed, particularly, in cases with intraocular or orbital invasion towards the exenteration and radiation therapy to eradicate the appropriate spot formation. However, systemic metastases already may have occurred in these patients with advanced disease. Primary intraocular uveal melanoma in stage T4 infiltrating orbit may also infiltrate a conjunctival tissue. In the last decades the management of patients with uveal melanoma has changed towards globe sparing techniques. Stereotactic radiosurgery has recently been proposed as an alternative treatment for posterior uveal melanoma. Secondary infiltration of the conjunctiva after radiotherapy due to scleral infiltration is very rare $(19,20,21)$.

\section{References}

1. Jakobiec FA, Folberg R, Iwamoto T. Clinicopathologic characteristics of premalignant and malignant melanocytic lesions of the conjuctiva. Ophthalmology 1989; 96: 147-166.

2. Damato B, Coupland SE. Clinical mapping of conjunctival melanomas. Br J Ophthalmol 2008; 92: 1545-1549.

3. Damato B, Coupland SE. Management of conjunctival melanoma. Expert Rev. Anticancer Ther 2009; 9: 1227-1239.

4. Yanoff M, Fine BS. Ocular pathology. 5th Ed. Philadelphia: Mosby, 2002.

5. Furdova A, Pesko K, Strmen $P$ et al. Conjunctival nevus and melanoma. Bratislava Medical Journal 2007; 108: 287-291.

6. Folberg R, McLean IW, Zimmerman LE. Conjunctival melanosis and melanoma. Ophthalmology 1984; 91: 673-678.

7. Shields CL, Demirici H, Karatza E et al. Clinical survey of 1643 melanocytic and nonmelanocytic tumors of the conjunctiva. Ophthalmology 2004; 111: 1747-1754.

8. Char DH. Conjunctival malignancies: Diagnosis and management. In: Char DH (Ed). Clinical ocular oncology. New York: Churchill Livingstone; 1989.

9. Tuomala S, Kivela T. Metastatic pattern and survival in disseminated conjunctival melanoma: implications for sentinel lymph node biopsy. Ophthalmology 2004; 111: 816-821.
10. Schallenberg M, Niederdräing N, Steuhl KP et al. Topisches Mitomycin C als Therapie konjunktivaler Tumore. Ophthalmologe 2008; 105: 777-784.

11. Stannard CE, Sealy GR, Hering ER et al. Malignant melanoma of the eyelid and palpebra; conjunctiva treated with iodine/125 brachytherapy. Ophthalmology 2000; 107: 951-958.

12. Herold TR, Hintschich $\mathbf{C h}$. Interferon $\alpha$ for the treatment of melanocytic conjunctival lesions. Graefes Arch Clin Exp Ophthalmol 2010; 248: 111-115.

13. Kurli M, Finger PT. Topical mitomycin chemotherapy for conjunctival malignant melanoma and primary acquired melanosis with atypia: 12 years` experience. Graefes Arch Clin Exp Ophthalmol 2005; 243: 1108-1114.

14. Shields JA, Shields CL, Mashayekhi A et al: Primary Acquired Melanosis of the Conjunctiva: Risk for Progression to Melanoma in 311 Eyes. Ophthalmology 2008; 115: 511-519.

15. Chalasani R, Giblin M, Conway RM. Role of topical chemotherapy for primary acquired melanosis and malignant melanoma of the conjunctiva and cornea: review of the evidence and recommendations for treatment. Clin Exp Ophthalmol 2005; 34: 708-714.

16. Kim JW, Abramson DH. Topical treatment options for conjunctival neoplasms. Clin Ophthalmol 2008; 2: 503-515.

17. Demirci H, McCormick SA, Finger PT. Topical mitomycin chemotherapy for conjunctival malignant melanoma and primary acquired melanosis with atypia: clinical experience with histopathologic observations. Arch Ophthalmol 2000; 118: 885-891.

18. Shields CL, Fasiudden A, Mashayekhi A et al. Conjunctival nevi: clinical features and natural course in 410 consecutive patients. Arch Ophthalmol 2004; 122: 167-175.

19. Furdova A, Chynoransky M, Krajcova P. Orbital melanoma. Bratisl Lek Listy 2011; 112 (8): 466-8.

20. Furdova A, Strmen P, Waczulikova I, Chorvath M, Sramka M, Slezak P. One-day session LINAC-based stereotactic radiosurgery of posterior uveal melanoma. Eur J Ophthalmol. 2011 Apr 18:0. doi: 10.5301/ EJO.2011.7733.

21. Furdova A, Slezak P, Chorvath M, Waczulikova I, Sramka M, Kralik G. No differences in outcome between radical surgical treatment (enucleation) and stereotactic radiosurgery in patients with posterior uveal melanoma. Neoplasma. 2010; 57(4): 377-381.

Received January 18, 2011. Accepted March 22, 2012. 